\title{
Association of genetic loci for migraine susceptibility in the she people of China
}

Qi-Fang Lin ${ }^{1}$, Xian-Guo Fu², Long-Teng Yao', Jing Yang ${ }^{2}$, Luo-Yuan Cao², Yong-Tong Xin ${ }^{1}$, Jun-Xia Hou', Lin-Feng $\mathrm{Ye}^{1}$ and Gen-Bin Huang ${ }^{1 *}$

\begin{abstract}
Background: The purpose of this study was to investigate the association of the genotype and allele frequencies of the polymorphisms rs4379368, rs10504861, rs10915437, rs12134493 and rs13208321 in She people of China with migraine headache susceptibility. The five alleles were previously identified as being associated with migraine in a Western population, but it was not known if this association would hold in a She population. rs4379368 is in the succinic HMG coenzyme A transferase (C7orf10) gene; rs10504861 is near the matrix metallopeptidase 16 (MMP16) gene; rs10915437 is near the adherens junctions associated protein 1 (AJAP1) gene; rs12134493 is upstream of the tetraspanin 2 (TSPAN2) gene; and rs13208321 is within the four and a half LIM domains protein 5 (FHL5) gene.
\end{abstract}

Methods: This was a case-controlled study conducted in She people of Fujian province in China. Polymerase chain reaction-restriction fragment length polymorphism and direct sequencing were performed. Univariate and multivariate analyses were used to assess the association of the different genotypes of each SNP with migraine.

Results: The rs4379368 T allele was not in Hardy-Weinberg equilibrium and was more common than the $C$ allele in subjects with migraine (58.7\%; $P=0.049$ ), possibly suggesting a selection bias for T allele in this population. In support of this, the $C T$ and $\Pi$ genotypes were more frequent in the migraine compared with the control groups (54.0 \% and $31.7 \%$ vs. $48.0 \%$ and $28.7 \%$, respectively; $P=0.019$ ). These genotypes were also more common in females with migraines than females without migraines (53.8 \% and $30.9 \%$ vs. $46.7 \%$ and $27.6 \% ; P=0.026$ ). Univariate and multivariate analyses found the CC genotype of rs4379368 and AA or AG genotype of rs13208321 were associated with a reduced risk of migraine ( $P$ values $\leq 0.039$ ).

Conclusions: Our findings suggest that rs4379368 and rs13208321 are potential genetic markers for migraine in this She population. The findings of this study and others indicate important differences between ethnic populations in regard to genetic markers of migraine susceptibility.

Keywords: Migraine; Single-nucleotide polymorphism; Susceptibility; She people; rs13208321; rs13208321

\section{Background}

Migraine headache refers to a common, chronic, and recurrent headache that significantly impacts the ability to work, results in greater health care resource utilization and cost, and a reduced quality of life $[1,2]$. A survey study on migraine, conducted in USA in 2010, showed the prevalence of migraine was $13.2 \%$ in the total population, $8.6 \%$ in males, and $17.5 \%$ in females [3].

\footnotetext{
* Correspondence: 2388988966@qq.com

'Department of Internal Neurology, Ningde Municipal Hospital, Affilliated Hospital of Fujian Medical University, Jiaocheng District, Ningde City, Fujian 352100, China

Full list of author information is available at the end of the article
}

The etiology and pathogenesis of migraine are still poorly understood [4]. Epidemiological studies found that there is a genetic and familial predisposition for migraine [5-7]. We previously performed an epidemiological study in the She ethnic minority population from Fujian province in China [5]. She people have their own special lifestyle, cultural heritage, and genetic background, which are different from those of Han people and other Asians. The study was a cross-sectional survey in which we evaluated the prevalence of migraine within the She population. We found the prevalence of migraine was about $10.5 \%$.

\section{实 Springer}


Recently, common genetic variants were identified that are associated with migraine susceptibility through twin, family clustering, and genome wide association studies of patients with migraine (with and without aura) [8, 9]. Several genome-wide association studies have investigated risk variants associated with migraines [10, 11]. Recently, Anttila et al. [11] conducted a large migraine meta-analysis that included 29 studies and 23285 patients with migraines and 95425 controls. They identified 12 single nucleotide polymorphisms (SNPs) that were associated with migraine susceptibility. Five of these SNPs had never previously been associated with the condition. They are: rs4379368 located in an intron of the succinic HMG coenzyme A transferase (C7orf10) gene; rs10504861 located close to the matrix metallopeptidase 16 (MMP16) gene; rs10915437 positioned in the vicinity of the adherens junctions associated protein 1 (AJAP1) gene; rs12134493 located upstream of the tetraspanin 2 (TSPAN2) gene; and rs13208321 located within the four and a half LIM domains protein 5 (FHL5) gene. To date only one study performed in a Spanish population has further tested the association of these five polymorphisms with susceptibility and/or pathogenesis of migraine [12], and no studies have evaluated these polymorphisms in a Chinese population.

The purpose of the current study was to evaluate the association of the newly identified five loci from the study of Anttila et al. with migraine in a She population. We chose these five alleles as there is no information regarding their association with migraine in non-Western ethnic backgrounds, and this information may help to give insight into what polymorphisms may influence migraine independent or dependent upon ethnicity.

\section{Methods}

\section{Subjects}

The study recruited subjects $(N=600)$ from the population of our prior study [5]. Migraine was diagnosed according to the Diagnostic criteria for Migraine developed by the Headache Classification Committee of the International Headache Society in 2004 [13]. Control subjects $(n=300)$ had no personal history or family history of chronic headache. Patients $(n=300)$ and controls were matched in age and gender. This study was approved by the Ethics Committee of our hospital (The IRB of Ningde Municipal Hospital, Affilliated Hospital of Fujian Medical University) and written informed consent was obtained from each patient. The IRB of Ningde Municipal Hospital, Affilliated Hospital of Fujian Medical University.

\section{Polymorphism analysis}

Peripheral venous blood (1-3 mL) was collected and whole blood genomic DNA was extracted and stored at $-20{ }^{\circ} \mathrm{C}$.
Polymerase chain reaction-restriction fragment length polymorphism (PCR-RFLP) was used to detect the genotypes of rs4379368, rs10504861 and rs10915437, and direct sequencing was used to evaluate rs12134493 and rs13208321. The findings of the PCR-RFLP were confirmed by directly sequencing randomly selected samples, which was performed by Fuzhou Di'en Biotech Co., Ltd. (Fujian, China). The possible genotypes or rs12134493 were possible AA, AC and CC, and for rs13208321 were AA, AG and GG.

The primers used for the polymerase chain reaction (PCR) were designed by our group and synthesized in Shanghai Sangon Biotech Co., Ltd. (Shanghai, China). Table 1 presents the sequence of different primers used for amplification of DNA, PCR-RFLP, and sequencing analysis. PCR analysis used the following conditions: denaturation at $94{ }^{\circ} \mathrm{C}$ for $5 \mathrm{~min}, 30$ cycles of denaturation at $94{ }^{\circ} \mathrm{C}$ for $30 \mathrm{~s}$, annealing at $58{ }^{\circ} \mathrm{C}$ for $45 \mathrm{~s}$ and extension at $72{ }^{\circ} \mathrm{C}$ for $60 \mathrm{~s}$, and a final extension at $72{ }^{\circ} \mathrm{C}$ for $5 \mathrm{~min}$. The reaction mixture for PCR $(25 \mu \mathrm{L})$ included $\mathrm{ddH}_{2} \mathrm{O}(18 \mu \mathrm{L})$, $10 \times$ Buffer $(2.5 \mu \mathrm{L}), 2.5 \mathrm{mM}$ dNTP $(2 \mu \mathrm{L}), 50 \mu \mathrm{M} / \mu \mathrm{L}$ forward primer $(0.5 \mu \mathrm{L}), 50 \mu \mathrm{M} / \mu \mathrm{L}$ reverse primer $(0.5 \mu \mathrm{L}), 5$ units $/ \mu \mathrm{L}$ Taq polymerase $(0.25 \mu \mathrm{L})$ and $50 \mathrm{ng} / \mu \mathrm{L}$ DNA template $(2 \mu \mathrm{L})$. Following amplification, $5 \mu \mathrm{L}$ of PCR product was analyzed by $1 \%$ agarose gel electrophoresis.

Products from PCR for rs4379368, rs10504861 and rs10915437 were subjected to incubation with restriction endonuclease to detect the presence of the different polymorphisms. The reaction mixture $(20 \mu \mathrm{L})$ included $\mathrm{ddH}_{2} \mathrm{O}(7.5 \mu \mathrm{L})$, products from PCR $(10 \mu \mathrm{L})$, Buffer $(2 \mu \mathrm{L})$, restriction endonuclease $(0.5 \mu \mathrm{L})$, either $\mathrm{HphI}$ or Tail (Thermo Scientific, Waltham, MA, USA) or MspA1I (New England Biolabs, Ipswich, MA, USA). Incubation was done at $37^{\circ} \mathrm{C}$ for $8-16 \mathrm{~h}$. Incubation with Tail restriction endonuclease was done for $8-16 \mathrm{~h}$ at $65{ }^{\circ} \mathrm{C}$. The digested products were separated by agarose electrophoresis. The specific possible restriction patterns are presented in Additional file 1.

Table 1 Primers used to expansion of 5 polymorphic sites by PCR

\begin{tabular}{lll}
\hline Polymorphisms & Primers & Sequence \\
\hline rs4379368 & Forward & 5'-AGTGGGCTTTCATTCTGGAA-3' \\
& Reverse & 5'-AAGGGCCTGAAATCTAATTCC-3' \\
rs10504861 & Forward & 5'-CGTAAGAGTAATATATTGGCCCA-3' \\
& Reverse & 5'-TTCTCAATTTTGTGGTTACATGC-3' \\
rs10915437 & Forward & 5'-TCCTTAAGGTTCTGGCTGGGT-3' \\
& Reverse & 5'-TGGCCCTCACTAAGGGTGATT-3' \\
rs12134493 & Forward & 5'-TTGTTTTCTGTGCCCGACAT-3' \\
& Reverse & 5'-GAGGAAATAGAAGTGTGGGGA-3' \\
rs13208321 & Forward & 5'-CGCCATTTCAGAGCTGTCA-3' \\
& Reverse & 5'-TTTTGGTCATGTCCCTTCCT-3' \\
\hline
\end{tabular}




\section{Statistical analyses}

Categorical variables were presented as numbers and percentages, and continuous variables were shown as mean and standard deviation. $T$-test was used to compare age difference between migraine and control groups. Chi-square goodness-of-fit tests were performed to test violation of Hardy-Weinberg equilibrium by comparing observed numbers to expected values of the three genotypes for each polymorphism. Univariate logistic regression was performed to examine the association between the genotypes of the different SNPs and migraine status. Multiple logistic regression analysis was performed by adjusting for age and gender. In addition, the migraine group was further divided into subgroups according to clinical characteristics of migraine. The distributions of genotype frequencies were compared between the genotypes of the SNPs and migraine-related characteristics. Post-hoc power analysis revealed the power was $0.99,0.89,0.98$ and 0.94 for detecting the statistical significance of associations between migraine status and genotypes of rs4379368, rs10915437, rs12134493 and rs13208321, respectively. All statistical analyses were carried out with IBM SPSS statistical software version 22 for Windows (IBM Corp., New York, USA). Statistical significance was defined as $P$ value $<0.05$.

\section{Results}

\section{Study subjects}

The demographics of the subjects were similar between groups (Table 2). The mean age was about 45 years and the majority of subjects were female ( $\geq 70 \%)$.

\section{Hardy-Weinberg equilibrium}

Not all of the SNPs were found to be in Hardy-Weinberg equilibrium. The genotypes of SNPs rs4379368 $(P=0.049)$ and rs10504861 $(P=0.007)$ in the migraine group were out of Hardy-Weinberg equilibrium (Table 3). The genotypes of rs10504861 were also out of Hardy-Weinberg equilibrium in the control group $(P=0.018)$; consequently, this SNP was excluded from subsequent statistical analyses.

\section{Allele frequency in migraine and control groups}

The frequency of $\mathrm{T}$ allele of rs4379368 was significantly higher in the migraine compared with the control group

Table 2 Description of sample demographic characteristics

\begin{tabular}{|c|c|c|c|c|c|}
\hline & \multicolumn{2}{|c|}{ Migraine $(n=300)$} & \multicolumn{2}{|c|}{ Control $(n=300)$} & \multirow[b]{2}{*}{$P$ value } \\
\hline & $\bar{n}$ & $\%$ & $\bar{n}$ & $\%$ & \\
\hline Gender & & & & & 0.236 \\
\hline Male & 77 & 25.7 & 90 & 30.0 & \\
\hline Female & 223 & 74.3 & 210 & 70.0 & \\
\hline $\mathrm{Age}^{\mathrm{a}}$ & 46.4 & 13.0 & 44.4 & 13.4 & 0.064 \\
\hline
\end{tabular}

${ }^{\mathrm{a}}$ Shown as mean and standard deviation
Table 3 Allele frequencies and testing for Hardy-Weinberg equilibrium of selected SNP markers

\begin{tabular}{|c|c|c|c|c|c|}
\hline \multirow[b]{2}{*}{ SNP } & \multirow[b]{2}{*}{ Allele ${ }^{a}$} & \multicolumn{2}{|l|}{ Migraine } & \multicolumn{2}{|l|}{ Control } \\
\hline & & $\overline{M A F^{b}}$ & $P$ value & $\overline{M A F^{b}}$ & $P$ value \\
\hline s4379368 & $\mathrm{C} / \mathrm{T}(\mathrm{T})$ & $352(58.7 \%)$ & $0494^{c}$ & $316(52.7 \%)$ & 0.5187 \\
\hline s10504861 & $\mathrm{C} / \mathrm{T}(\mathrm{C})$ & 535 (89.2 \%) & $0.0074^{c}$ & $538(89.7 \%)$ & $0.0180^{\circ}$ \\
\hline rs10915437 & $\mathrm{A} / \mathrm{G}(\mathrm{A})$ & $396(66.0 \%)$ & 0.4905 & 407 (67.8 \%) & 0.1148 \\
\hline 1512134493 & $\mathrm{~A} / \mathrm{C}(\mathrm{C})$ & 6) & 0. & 5 & 0. \\
\hline s13208321 & $\mathrm{A} / \mathrm{G}(\mathrm{G})$ & 584 (97.3\%) & 0.6351 & 569 (94.8\%) & 0.1577 \\
\hline
\end{tabular}

${ }^{a}$ Major allele was indicated in parenthesis

${ }^{\mathrm{b}} \mathrm{MAF}$ major allele frequency; both number of the major allele and percentage in the particular group were shown

'Violation of Hardy-Weinberg equilibrium

(58.7 \% vs. $52.7 \% ; P=0.036$ ) (Table 3 ). The $\mathrm{C}$ allele frequency was more frequent in the migraine then the control groups for rs12134493 (95.5\% vs. $93.5 \%$, respectively; $P=0.026$ ). Significant associations between allele frequencies and migraine status were found for rs12134493 among males $(P=0.041)$, and for rs4379368 $(P=0.042)$ and $r s 13209321(P=0.026)$ among females (data not shown).

\section{Distribution of genotypes of SNPs}

A greater percentage of subjects carried the CC genotype of rs4379368 in the control group than those in the migraine group ( $23.3 \%$ vs. $14.3 \%$, respectively). Univariate analysis found that participants carrying the CC genotype of rs4379368 were less likely to have migraine (odds ration $[\mathrm{OR}]=0.56$; $95 \%$ confidence interval $[\mathrm{CI}]$, 0.34-0.90; $P=0.016$ ) (Table 4). Mutlivariate analysis, which controlled for age and gender, also found rs4379368 was associated with a lower risk of migraine $(\mathrm{OR}=0.52 ; 95 \% \mathrm{CI}, 0.32-0.85 ; P=0.008)$. Univariate and multivariate analyses found that the AA and AG genotypes of rs13208321e were associated with lower odds of migraine ( $P$ values $\leq 0.047$ ), but found no increased or decreased risk of migraine with rs4379368 or rs13208321 genotypes.

\section{Comparisons of SNPs between migraine patients with or} without aura, and controls

Migraine patients were stratified based on the presence or absence of aura. Fewer people in the migraine without aura group carried the CC genotype of rs4379368 than those in the migraine with aura (22.6 vs. $12.2 \% ; P=$ $0.011)$ and control (23.3 vs. $12.2 \% ; P=0.003)$ groups (Table 5). Similarly, the C-allele frequency of rs4379368 was lower in the migraine without aura (38.4\%) compared with the migraine with aura (52.4\%) and control $(47.3 \%)$ groups $(P=0.005)$. No other SNP was associated with aura, and none of the SNPs were associated with additional features including family history, frequency, 
Table 4 Comparison of distribution of genotypes of selected SNP markers between migraine and control group

\begin{tabular}{|c|c|c|c|c|c|c|c|c|}
\hline \multirow[b]{3}{*}{ SNP } & \multicolumn{4}{|l|}{ All } & & & & \\
\hline & \multicolumn{2}{|c|}{ Migraine $(n=300)$} & \multicolumn{2}{|c|}{ Control $(n=300)$} & \multicolumn{2}{|c|}{ Univariate analysis } & \multicolumn{2}{|c|}{ Multivariate analysis $^{a}$} \\
\hline & $\mathrm{n}$ & $\%$ & $\mathrm{n}$ & $\%$ & OR (95\% Cl) & $P$ value & $\mathrm{OR}^{\mathrm{a}}(95 \% \mathrm{Cl})$ & $P$ value \\
\hline \multicolumn{9}{|l|}{ rs4379368 } \\
\hline CC & 43 & 14.3 & 70 & 23.3 & $0.56(0.34,0.90)$ & 0.016 & $0.52(0.32,0.85)$ & 0.008 \\
\hline CT & 162 & 54 & 144 & 48 & $1.02(0.71,1.47)$ & 0.923 & $0.98(0.68,1.43)$ & 0.932 \\
\hline$\pi$ & 95 & 31.7 & 86 & 28.7 & 1.00 & & 1.00 & \\
\hline \multicolumn{9}{|l|}{ rs10915437 } \\
\hline GG & 32 & 10.7 & 37 & 12.3 & $0.97(0.57,1.65)$ & 0.919 & $0.98(0.58,1.67)$ & 0.948 \\
\hline$A G$ & 140 & 46.7 & 119 & 39.7 & $1.32(0.94,1.86)$ & 0.107 & $1.35(0.96,1.91)$ & 0.086 \\
\hline AA & 128 & 42.7 & 144 & 48 & 1.00 & & 1.00 & \\
\hline \multicolumn{9}{|l|}{ rs12134493 } \\
\hline$A A$ or $A C$ & 26 & 8.6 & 39 & 13 & $0.64(0.38,1.07)$ & 0.090 & $0.64(0.38,1.08)$ & 0.092 \\
\hline CC & 274 & 91.3 & 261 & 87 & 1.00 & & 1.00 & \\
\hline \multicolumn{9}{|l|}{ rs13208321 ${ }^{c}$} \\
\hline$A A$ or $A G$ & 16 & 5.3 & 29 & 9.7 & $0.53(0.28,0.99)$ & 0.047 & $0.51(0.27,0.97)$ & 0.039 \\
\hline GG & 284 & 94.7 & 271 & 90.3 & 1.00 & & 1.00 & \\
\hline
\end{tabular}

$O R$ odds ratio, $\mathrm{Cl}$ confidence interval

${ }^{a} \mathrm{Age}$ and gender were included as covariates

${ }^{\mathrm{b}}$ Genotyp $A A$ and $A C$ were combined into one group due to small sample size

${ }^{\mathrm{c}} \mathrm{Genotype} A \mathrm{~A}$ and $\mathrm{AG}$ were combined into one group due to small sample size

duration, laterality, pulsing pain, nausea, photophobia, phonophobia, or physical activity (Additional file 1: Table S1).

\section{Discussion}

Our study evaluated the association of the newly identified rs4379368, rs10504861, rs10915437, rs12134493, and rs1320821 polymorphisms with migraine headache in a She population from the Fujian province in China. The alleles of rs4379368 were not in Hardy-Weinberg equilibrium in the migraine groups and the $\mathrm{T}$ allele of rs4379368 was more common than the $\mathrm{C}$ allele in subjects with migraine, possibly suggesting a selection bias for this allele in this population. In support of this, the $\mathrm{CT}$ and TT genotypes were more frequent in the migraine compared with the control groups $(P=0.019)$, while the presence of the $\mathrm{CC}$ genotype reduced the risk of migraine. These $\mathrm{CT}$ and TT genotypes were also more common in females with migraines than females without migraines. There was no difference in the frequency of the rs4379368 genotypes in males. These findings suggest that carrying the $\mathrm{T}$ allele of rs4379368 and being female puts you at risk for migraine headaches in this She population. Subgroup analysis indicated that subjects without aura had a lower frequency of the CC genotype than subjects with migraines with aura or healthy controls, suggesting genetic differencing in regard to migraine type. Univariate and multivariate analysis found that the AA and AG genotypes rs13208321 were associated with lower risk of migraine than the other alleles. However, none of the genotypes of this SNP were associated with migraine-related characteristics.

The alleles of rs105048561 were also not in HardyWeinberg equilibrium in either the migraine or control groups. The $\mathrm{C}$ allele was the most common allele in both groups. As this is the first study to describe the genetic properties of the rs105048561 loci, it is not known if this lack of Hardy-Weinberg equilibrium for rs10504856 is specific to the She population or is more general across other ethnic populations in China or other parts of the world. Additional studies are required to address this issue. For the other three alleles studied, there was no difference in allelic or genotypic frequency between the migraine and control groups.

A study by Chasman et al. [14] found that migraines can be subdivided not only be the presence or absence of an aura but also by a number of other features in including family history, frequency, duration, laterality, pulsing pain, nausea, photophobia, phonophobia, or aggreavation by physical activity. Other than the association of rs4379368 CC genotype with migraine with aura, none of the SNPs were associated with these other migraine-associated characteristics.

\section{Comparison of findings with prior studies}

Our findings differ from the study of Anttila et al. [11] which found all five of the SNPs we investigated were 
Table 5 Comparisons of migraine with or without aura, and controls

\begin{tabular}{|c|c|c|c|c|c|c|}
\hline \multirow[b]{3}{*}{ SNP } & \multicolumn{6}{|c|}{ Migraine } \\
\hline & \multicolumn{2}{|c|}{ Aura $(n=62)$} & \multicolumn{2}{|c|}{ Without aura $(n=238)$} & \multicolumn{2}{|c|}{ Control $(n=300)$} \\
\hline & $\mathrm{n}$ & $\%$ & $\mathrm{n}$ & $\%$ & $\mathrm{n}$ & $\%$ \\
\hline \multicolumn{7}{|c|}{ rs4379368 } \\
\hline CC & 14 & 22.6 & 29 & $12.2^{\mathrm{a}}$ & 70 & $23.3^{b}$ \\
\hline CT & 37 & 59.7 & 125 & 52.5 & 144 & 48.0 \\
\hline$\pi$ & 11 & 17.7 & 84 & 35.3 & 86 & 28.7 \\
\hline \multicolumn{7}{|c|}{ rs10504861 } \\
\hline CC & 56 & 90.3 & 187 & 78.6 & 245 & 81.7 \\
\hline CT & 4 & 6.5 & 45 & 18.9 & 48 & 16.0 \\
\hline$\Pi$ & 2 & 3.2 & 6 & 2.5 & 7 & 2.3 \\
\hline \multicolumn{7}{|c|}{ rs10915437 } \\
\hline AA & 26 & 41.9 & 102 & 42.9 & 144 & 48.0 \\
\hline$A G$ & 30 & 48.4 & 110 & 46.2 & 119 & 39.7 \\
\hline GG & 6 & 9.7 & 26 & 10.9 & 37 & 12.3 \\
\hline \multicolumn{7}{|c|}{ rs12134493 } \\
\hline AA & 0 & 0.0 & 1 & 0.4 & 0 & 0.0 \\
\hline$A C$ & 6 & 9.7 & 19 & 8.0 & 39 & 13.0 \\
\hline CC & 56 & 90.3 & 218 & 91.6 & 261 & 87.0 \\
\hline \multicolumn{7}{|c|}{ rs13208321 } \\
\hline $\mathrm{AA}$ & 0 & 0.0 & 0 & 0.0 & 2 & 0.7 \\
\hline$A G$ & 1 & 1.6 & 15 & 6.3 & 27 & 9.0 \\
\hline GG & 61 & 98.4 & 223 & 93.7 & 271 & 90.3 \\
\hline
\end{tabular}

${ }^{\mathrm{a}} P<0.05$ for Aura vs. Non-aura

${ }^{\mathrm{b}} P<0.05$ for Non-aura vs. control

associated with risk of migraine in a Western population. Our results also differ from those of Sintas et al. [12] that assessed the association of a subset of SNPs identified by Antilla et al. with migraine in a Spanish population. Sintas et al. evaluated 12 SNPs in a population of people who had migraine with aura $(n=512)$ and migraine-free controls $(n=535)$. They found a nominal association of four SNPs with migraine with aura: rs2651899 (within the PR domain containing 16 [PRDM16] gene), rs10166942 (near TRPM8), rs12134493 and rs10504861. In contrast to our findings, they saw no association of rs4379368 with migraine $(P$ values $>0.226)$. In addition, we found no association of rs1213449 or rs10504861 with migraine. Our study did not evaluate the association of rs2651899 or rs10166942 with migraine.

The difference in findings between our study and the two prior studies may result from ethnic/genetic differences between the study populations. Alternatively, the differences between the studies may reflect the small sample size of our study.

As mentioned previously, Anttila et al. found an association of rs4379368 and rs13208321with migraine [11].
Of note, the $\mathrm{C}$ allele of rs4379368 and the $\mathrm{T}$ allele of rs10504861 are the major alleles for these loci in Western countries [11], while in our population they are the minor alleles. Our study and that of Anttila et al. support the idea that rs4379368 and rs13208321 are genetic markers for migraine. The rs4379368 SNP is located within an intron in the transcript of the C7orf10 gene, which encodes succinic HMG coenzyme A transferase [15]. The various alleles might differentially influence the transcription and/or RNA processing of the gene and consequently affect the risk of migraine. rs13208321 is located within the FHL5 gene and may influence protein function.

The SNP rs12134493, located upstream of the TSPAN2 gene, was not associated with migraine in our total study population but the CC genotype was more frequent in males with migraine compared with those without migraine. TSPAN2 encodes a four transmembrane protein in cells which mediates signal transduction and is involved in the modulation of development, activation, growth, and movement of cells [16]. The role of the TSPAN2 region of the genome in migraine is supported by the results of another genome-wide study by Esserlind et al. [17] that found another SNP (rs2078371) located near TSPAN2 reached genome-wide significance for association with migraine in Danish and Icelandic populations. Our study and that of Esserlind et al. evaluated different sets of SNPs; Esserlind et al. assessed the top six SNPs identified in a prior genome-wide study migraine association study which did not include any of the SNPs we investigated [10]. The potential role of TSPAN2 in migraine headaches is unclear. Larger multi-centered clinical studies with greater sample sizes in geographically and genetically diverse populations are required to elucidate the role of these SNPs in migraine headache susceptibility.

\section{Conclusion}

Several large genome-wide association studies have identified genes that are possibly associated with the susceptibility of migraine headaches [10, 11, 18-21]. We investigated the relationship of five SNPs with susceptibility of migraine headaches in Chinese She subjects. Our findings suggest that carrying the $\mathrm{T}$ allele of rs4379368 and being female puts you at risk for migraine headaches in this She population. Our findings support the idea that rs4379368 and rs13208321 may be genetic marker for susceptibility for migraine headaches.

\section{Additional file}

Additional file 1: Restriction pattern of different PCR products. Table

S1. Comparisons of migraine with or without aura, and controls. 


\section{Abbreviations}

SNP: Single nucleotide polymorphisms; C7orf10: Succinic HMG coenzyme A transferase gene; MMP16: Metallopeptidase 16 gene; AJAP1: Adherens junctions associated protein 1 gene; TSPAN2: Tetraspanin 2 gene; FHL5: Four and a half LIM domains protein 5 gene; PCR-RFLP: Polymerase chain reaction-restriction fragment length polymorphism; PCR: Polymerase chain reaction; bp: Base pairs; PRDM16: PR domain containing 16 gene; TRMP8: Transient receptor potential cation channel subfamily M member 8 .

\section{Competing interests}

The authors declare that they have no competing interests.

\section{Authors' contributions}

Q-FL carried out study design, experimental studies, participated in manuscript editing; X-GF participated in data analysis; L-TY participated in clinical studies; JY participated in literature research; L-YC participated in manuscript preparation; Y-TX participated in clinical studies; J-XH participated in statistical analysis; L-FY participated in statistical analysis; $\mathrm{G}-\mathrm{bH}$ is the guarantor of integrity of the entire study and participated in study design, manuscript review. All authors read and approved the final manuscript.

\section{Acknowledgments}

The authors sincerely thank the She ethnic minority group for their help and willingness to participate in this study.

\section{Financial support}

This work was supported by grant 2014-CXB-26 from the Fujian Medical Innovating Program.

\section{Author details}

'Department of Internal Neurology, Ningde Municipal Hospital, Affilliated Hospital of Fujian Medical University, Jiaocheng District, Ningde City, Fujian 352100, China. ${ }^{2}$ Laboratory of Cell and Molecular Biology, Ningde Municipal Hospital, Affilliated Hospital of Fujian Medical University, Ningde, Fujian, China.

\section{Received: 8 April 2015 Accepted: 6 July 2015}

Published online: 01 August 2015

\section{References}

1. Adams AM, Serrano D, Buse DC, Reed ML, Marske V, Fanning KM, Lipton RB (2015) The impact of chronic migraine: The Chronic Migraine Epidemiology and Outcomes (CaMEO) Study methods and baseline results. Cephalalgia 35(7):563-78

2. Arslantas D, Tozun M, Unsal A, Ozbek Z (2013) Headache and its effects on health-related quality of life among adults. Turk Neurosurg 23(4):498-504

3. Victor TW, Hu X, Campbell JC, Buse DC, Lipton RB (2010) Migraine prevalence by age and sex in the United States: a life-span study. Cephalalgia 30(9):1065-1072

4. Paemeleire K, Louis P, Magis D, Vandenheede M, Versijpt J, Vandersmissen B, Schoenen J (2015) Diagnosis, pathophysiology and management of chronic migraine: a proposal of the Belgian Headache Society. Acta Neurol Belg 115(1):1-17

5. Huang GB, Yao LT, Hou JX, Zhang ZJ, Xin YT, Wu XY, Lu GY, Chen ZQ, Huang JP (2013) Epidemiology of migraine in the She ethnic minority group in Fujian province, China. Neurol Res 35(7):684-692

6. Peng KP, Wang SJ (2014) Epidemiology of headache disorders in the Asia-pacific region. Headache 54(4):610-618

7. Seyed Saadat SM, Hosseininezhad M, Bakhshayesh B, Hoseini M, Naghipour M (2014) Epidemiology and clinical characteristics of chronic daily headache in a clinic-based cohort of Iranian population. Neurol Sci 35(4):565-570

8. Girotra P, Singh SK, Saini D (2014) Disentangling the intricacies of migraine: a review. CNS Neurol Disord Drug Targets 13(5):776-791

9. Gasparini CF, Sutherland HG, Griffiths LR (2013) Studies on the pathophysiology and genetic basis of migraine. Curr Genomics 14(5):300-315

10. Chasman DI, Schurks M, Anttila V, de Vries B, Schminke U, Launer $L$, Terwindt GM, van den Maagdenberg AM, Fendrich K, Volzke H, Ernst F, Griffiths LR, Buring JE, Kallela M, Freilinger T, Kubisch C, Ridker PM, Palotie A, Ferrari MD, Hoffmann W, Zee RY, Kurth T (2011) Genome-wide association study reveals three susceptibility loci for common migraine in the general population. Nat Genet 43(7):695-698

11. Anttila V, Winsvold BS, Gormley P, Kurth T, Bettella F, McMahon G, Kallela M, Malik R, de Vries B, Terwindt G, Medland SE, Todt U, McArdle WL, Quaye L, Koiranen M, Ikram MA, Lehtimäki T, Stam AH, Ligthart L, Wedenoja J, Dunham I, Neale BM, Palta P, Hamalainen E, Schürks M, Rose LM, Buring JE, Ridker PM, Steinberg S, Stefansson H et al (2013) Genome-wide meta-analysis identifies new susceptibility loci for migraine. Nat Genet 45(8):912-917. doi:10.1038/ ng.2676, Epub 2013 Jun 23

12. Sintas C, Fernandez-Morales J, Vila-Pueyo M, Narberhaus B, Arenas C, Pozo-Rosich P, Macaya A, Cormand B (2014) Replication study of previous migraine genome-wide association study findings in a Spanish sample of migraine with aura. Cephalalgia. [Epub ahead of print].

13. Headache Classification Subcommittee of the International Headache $\mathbf{S}$ (2004) The International Classification of Headache Disorders: 2nd edition. Cephalalgia 24(Suppl 1):9-160

14. Chasman DI, Anttila V, Buring JE, Ridker PM, Schurks M, Kurth T, International Headache Genetics Consortium (2014) Selectivity in genetic association with sub-classified migraine in women. PLoS Genet 10(5):e1004366

15. Marlaire S, Van Schaftingen E, Veiga-da-Cunha M (2014) C7orf10 encodes succinate-hydroxymethylglutarate CoA-transferase, the enzyme that converts glutarate to glutaryl-CoA. J Inherit Metab Dis 37(1):13-19

16. Lafleur MA, Xu D, Hemler ME (2009) Tetraspanin proteins regulate membrane type-1 matrix metalloproteinase-dependent pericellular proteolysis. Mol Biol Cell 20(7):2030-2040

17. Esserlind AL, Christensen AF, Le H, Kirchmann M, Hauge AW, Toyserkani NM, Hansen T, Grarup N, Werge T, Steinberg S, Bettella F, Stefansson H, Olesen J (2013) Replication and meta-analysis of common variants identifies a genome-wide significant locus in migraine. Eur J Neurol 20(5):765-772

18. Ghosh J, Pradhan S, Mittal B (2013) Genome-wide-associated variants in migraine susceptibility: a replication study from North India. Headache 53(10):1583-1594

19. lizuka T, Takahashi Y, Sato M, Yonekura J, Miyakawa S, Endo M, Hamada J, Kan S, Mochizuki H, Momose Y, Tsuji S, Sakai F (2012) Neurovascular changes in prolonged migraine aura in FHM with a novel ATP1A2 gene mutation. J Neurol Neurosurg Psychiatry 83(2):205-212

20. Ishii M, Onaya T, Katoh H, Kiuchi Y, Kasai H, Kawamura M, Shimizu S (2012) Association between the G252A Tumor Necrosis Factor-beta Gene Polymorphism and Medication-Overuse Headache. J Clin Neurol 8(4):301-304

21. Millan-Guerrero RO, Baltazar-Rodriguez LM, Cardenas-Rojas MI, Ramirez-Flores M, Isais-Millan S, Delgado-Enciso I, Caballero-Hoyos R, Trujillo-Hernandez B (2011) A280V polymorphism in the histamine $\mathrm{H} 3$ receptor as a risk factor for migraine. Arch Med Res 42(1):44-47

\section{Submit your manuscript to a SpringerOpen ${ }^{\circ}$ journal and benefit from:}

- Convenient online submission

- Rigorous peer review

- Immediate publication on acceptance

- Open access: articles freely available online

- High visibility within the field

- Retaining the copyright to your article

Submit your next manuscript at $>$ springeropen.com 